\title{
Corpus Callosum Hematoma, as a Rare Complication of COVID-19
}

\author{
Mohammad Khair Hamad ${ }^{1}$, Dima Takruri ${ }^{2}$, Satya Patro ${ }^{1}$, Mhd Baraa Habib ${ }^{1}$, and \\ Vishwajit Verma ${ }^{1}$ \\ ${ }^{1}$ Hamad Medical Corporation \\ ${ }^{2}$ Primary Health Care Corporation
}

August 2, 2021

\begin{abstract}
A 46-year-old gentleman had a complicated course of COVID-19 pneumonia. Despite the recovery of the respiratory status, he developed corpus callosum hematoma and critical illness neuropathy/myopathy, the clinical situation became more complicated by developing pulmonary embolism that required anticoagulation. Fortunately, the patient made a good recovery.
\end{abstract}

\section{Corpus Callosum Hematoma, as a Rare Complication of COVID-19}

Mohammad Khair Hamad ${ }^{1}$, Dima Hamed Takruri ${ }^{2}$, Satya Narayana Patro ${ }^{3}$, Mhd Baraa Habib ${ }^{4}$, Vishwajit Verma $^{5}$

${ }^{1}$ Internal Medicine Department, Hamad Medical Corporation, Doha, Qatar.

${ }^{2}$ Family Medicine Department, Primary Health Care Corporation, Doha, Qatar.

${ }^{3}$ Radiology Department, Hamad Medical Corporation, Doha, Qatar.

${ }^{4}$ Intensive Care Department, Hamad Medical Corporation, Doha, Qatar.

Corresponding author: Mohammad Khair Hamad, Internal Medicine Department, Hamad Medical Corporation, Doha, Qatar. Email: Mhamad6@hamad.qa

ORCID: 0000-0001-9739-0073

\section{Funding}

This research did not receive any specific grant from funding agencies in the public, commercial, or not-forprofit sectors.

\section{Declaration of Competing Interest}

The authors report no conflict of interest.

\section{Consent}

Written informed consent was obtained from the patient.

Credit authorship contribution statement 
MKH: Clinical care, literature review and manuscript writing. DHT: Literature review and manuscript writing. SNP: radiology imaging. MBH: manuscript writing VV: mentorship, clinical care, literature review and manuscript revision.

\title{
Acknowledgment
}

The authors would like to thank the patient for allowing us to share his details with the medical community. Additionally, we acknowledge the Qatar National Library for funding the open access fees of this publication.

\begin{abstract}
A 46-year-old gentleman had a complicated course of COVID-19 pneumonia. Despite the recovery of the respiratory status, he developed corpus callosum hematoma and critical illness neuropathy/myopathy, the clinical situation became more complicated by developing pulmonary embolism that required anticoagulation. Fortunately, the patient made a good recovery.
\end{abstract}

\section{Keywords}

Covid -19 pneumonia, corpus callosum hematoma, Pulmonary embolism, anticoagulation.

\section{Abbreviations}

GCS: Glasco coma scale, CPAP: Continuous positive airway pressure, DVT: deep vein thrombosis, MRI: Magnetic resonance imaging, CT: Computed tomography.

\section{Key Clinical Message}

A rare site of intracranial bleeding - Corpus callosum bleeding- complicating the course of COVID-19 pneumonia. And the complex management of pulmonary embolism in presence of intracranial bleeding.

\section{Introduction}

SARS COV 2 is causing a worldwide pandemic that mainly affects the pulmonary system. However, other system involvement is being increasingly noticeable in COVID-19 patients.[1] Common Neurological manifestations are anosmia, dysgeusia, and headache. Other serious complications, like stroke, are less commonly described. Intracerebral haemorrhage is rarely associated with COVID-19.[2] We present a COVID-19 patient who has a complicated clinical course, with challenging management.

\section{Case Report}

A 46-year-old male patient, who is not known to have previous chronic medical conditions, presented to the emergency department complaining of feeling of hotness, cough, and loss of smell of 4 days duration. On presentation, he was conscious, alert, and oriented to time, place, and person. He was febrile (Temperature of $38.9 \mathrm{C}^{\circ}$ ), tachycardic with heart rate of $104 \mathrm{bpm}$, tachypnoeic with a respiratory rate of 28 breaths per minute, Blood Pressure was 107/81 mm Hg and O2 saturation on room air was $97 \%$.

On physical examination, pupils were equal in size and reaction to light. Chest auscultation showed clear breath sounds bilaterally with normal S1S2 and no murmurs. Abdomen was flat, soft, with no tenderness or guarding. Extremities: no bipedal enema, bilateral full and equal pulses. GCS 15/15, no neurologic deficits.

Blood investigations revealed WBC to be $5.7 \times 109 \mathrm{~L}$, with low lymphocytic count $0.7^{*} 10^{3}$ uL Haemoglobin $15.3 \mathrm{~g} / \mathrm{dl}$, platelet count $122 \times 10^{9} \mathrm{~L}$, Urea $3.04 \mathrm{mmol} / \mathrm{l}$, creatinine $89 \mathrm{umol} / \mathrm{L}$, CRP $140 \mathrm{mg} / \mathrm{L}$, and normal electrolytes.

Chest x-ray demonstrated multiple pneumonitic patches seen in both lung fields which were suggestive of viral pneumonia. Nasopharyngeal swab for COVID-19 PCR was positive. Based on the above-mentioned work up the patient was admitted as a case of COVID-19 pneumonia. His condition deteriorated the next day of admission, and he was more tachypnoeic requiring $\mathrm{O} 2$ supplementation by nasal cannula. 
He was transferred to Medical intensive care unit (MICU) for observation. As his oxygen requirements increased, he was started on none rebreathing mask (NRM) then switched into non-invasive ventilation (CPAP). Despite this, his condition deteriorated further that required endotracheal intubation and he was started on mechanical ventilation.

He received a treatment protocol for COVID-19 infection according to our hospital protocol in accordance with the international guidelines at that time, which was as the following:

1-Hydroxy-Chloroquine $400 \mathrm{mg}$ once daily X 10 days

2-Azithromycin $500 \mathrm{mg}$ X 7 days

3- Methyl prednisolone $40 \mathrm{mg}$ IV q12hr for 5 days.

4- Tocilizumab $600 \mathrm{mg}$ IV 2 total of 2 doses ( 8 days between the 2 doses)

He was kept on enoxaparin for DVT prophylaxis, and the dose was adjusted according to his clinical situation with monitoring of Anti Xa. Furthermore, he received convalescent plasma.

During this time, the platelet counts had dropped to as low as $92^{*} 10^{3}$ for 2 days then improved to normal levels. The INR was normal, and heparin induced thrombocytopenia (HIT) test was negative. No noticeable overt bleeding episodes.

He was kept on mechanical ventilation and the ventilator setting was adjusted. Proning was required three times and his condition was gradually improving. Sedation and muscle relaxation were tapered off and after 18 days he was successfully extubated.

After extubation, the patient was unable to move his four limbs, but he had intact level of consciousness. Physical examination revealed a power of $1 / 5$ in all muscle groups of upper and

lower limbs, bilateral lower limb wasting with hyporeflexia, no fasciculations, plantar reflex was negative, and sensation was intact.

As an evaluation of his quadriplegia, brain\& spinal cord MRI (Figure1) were done and showed late subacute hematoma involving the corpus callosum, with a background of numerous supra and infratentorial foci of microbleeds. Cervicodorsal spinal cord MRI was unremarkable. Accordingly, enoxaparin was stopped on the same day.

Neurosurgery team was consulted regarding any possible therapeutic intervention for the subacute callosal hematoma. Since the hematoma was not causing any mass effect and the fact that it was subacute with no associated IVH, no neurosurgical intervention would be of benefit.

Neurology team evaluation was suggesting critical illness neuropathy/myelopathy as a cause of the quadriplegia rather than the hematoma itself. Due to isolation precautions nerve conduction study nor electromyography could be done.

Next day, he was noted to have persistent tachycardia with HR ranging 100-120 BPM. CT pulmonary angiogram (Figure 2) was done and showed two pulmonary thromboembolisms. The pulmonary thromboembolism involved the anterior branch of the left main pulmonary artery extending to sub-segmental branches and the posterior basal segmental and sub-segmental branches of right lower lobe pulmonary artery.

Considering the intracranial bleeding, he was commenced on heparin infusion with close monitoring of the neuro vitals. Then, he was switched into therapeutic low molecular weight heparin. Follow up head CT scan (Figure 3) demonstrated the same subacute corpus callosum hematoma, with no acute changes or bleeding.

COVID-19 PCR swab was repeated twice and both results were negative.

Patient was tapered off $\mathrm{O} 2$ supplementation and he maintained an acceptable O2 saturation on ambient air. 
After discussing the case with haematology team, with consideration to the patient condition, the patient was given therapeutic dose enoxaparin subcutaneously for 3 months as a case of provoked pulmonary thromboembolism.

Extensive physiotherapy sessions were started with gradual and noticeable improvement in the muscle groups power.

Patient was being treated as an inpatient for 2 months, then continued physiotherapy in rehabilitation institute for another 1 month. On discharge, muscle power was $4 / 5 \&$ he was independent in most of the activities of daily living, with need for assistance in using stairs only.

On further follow up, patient continued to attend outpatient physiotherapy sessions on weekly basis. He completed 3 months enoxaparin for anticoagulation, no reported bleeding episodes.

The Patient is now functioning independently, and he returned to his work.

\section{Discussion}

A novel coronavirus was first identified in China causing human respiratory disease.[3] As the virus is spreading and causing a worldwide pandemic, our understanding of the disease spectrum is evolving.

Accumulating evidence shows that SARS-COV-2 is not limited to the respiratory system but also has a deleterious effect on other organs and systems like the cardiovascular, gastrointestinal, and nervous system. $[1,4]$

Both glial and neuronal cells express ACE2 receptors, making them susceptible to SARS-COV-2 attack. [5-7] The virus makes the access to the brain either by the cerebral circulation, where ACE2 receptors are expressed in the endothelial lining of the cerebral vessels, or the retrograde path from the olfactory bulb through the cribriform plate. [5-8]

Both central and peripheral nervous system manifestations have been reported either as COVID-19 specific or related to critical illness.[2] Disease spectrum may range from anosmia, ageusia, headache and dizziness without other clinical features to more severe involvement like encephalitis, acute disseminated encephalomyelitis, myelitis, cerebrovascular manifestations, peripheral and muscle disease.[2]

Cerebrovascular disease is an important complication of COVID-19, with a reported incidence of 2-6\% of hospitalized patients. The hypercoagulable state with the inflammatory cascade leading to endothelial damage predispose to acute cerebrovascular events. [2]

Intracerebral haemorrhage is not a common event in general intensive care unit (ICU) patients. Hematologic malignancies, severe thrombocytopenia, sepsis complicated by renal and hepatic dysfunction, mechanical ventilation with high inspiratory pressures, and high $\mathrm{CO} 2$, all could be associated with increased risk of intracranial haemorrhage (ICH) in critical illness. [9]

Few case reports and case series reported intracranial haemorrhage as a complication of COVID-19, [4,10-18] while only one report showed that ICH happened before the respiratory manifestations.[4]

The presence of ACE2 receptors in the endothelium of cerebral vessels, makes them a target for SARSCOV-2, which leads to endothelial dysfunction and dysregulation of local blood pressure and flow, resulting in vessel wall rupture. [12-13,17] In a series of COVID-19 non survivors, post-mortem brain MRI suggests vasculopathic changes that could be related to the viral damaging effect on the endothelium.[19]

In some reports, the intracerebral bleeding was secondary to the haemorrhagic transformation of cerebral stroke, [12] haemorrhagic transformation of cerebral venous thrombosis, [18] meningoencephalitis complicated with ICH.[15]

In one small series of 3 patients, the cause of ICH was uncertain either secondary to therapeutic anticoagulation or as a complication of COVID-19. They also shared a common finding on brain imaging showing anoxic brain injury.[20] 
Diffuse brain oedema and multifocal haemorrhages support the speculation that anoxic brain injury and cytokine storm rather than anticoagulation led to ICH in COVID-19 patients. [6-7,10] In case series of COVID-19 non survivors, brain post-mortem histopathological examination revealed hypoxic injury without evidence of encephalitis.[21]

Spontaneous Corpus callosum hematoma is rarely described in literature.[22] Possible causes are traumatic brain injury, hypertension, ruptured anterior communicating artery or peri callosal artery aneurysm, bleeding associated with tumours or encephalitis,[23] which was not the case in our patient who did not have any of the risk factors.

One series of 11 patients describes diffuse leukoencephalopathy with micro-haemorrhages. In 4/11 patients the location of the micro haemorrhages was in the corpus callosum, all patients were on mechanical ventilation and on monitored anticoagulation, but no bleeding elsewhere in the body, brain hypoxia was proposed as the mechanism of the brain findings.[14]

SARS-COV-2 does not appear to be thrombogenic by itself. Rather, the coagulation abnormalities are secondary to the intense inflammatory reaction. The abnormal coagulation profile early in the infection is not translated to clinical bleeding as compared to other RNA viruses causing haemorrhagic fevers. [24]

The risk of venous thromboembolism (VTE) in critically ill patients due to COVID-19 is higher compared to the general ICU population, and the risk of VTE is still there even with prophylactic dose of anticoagulation. [24-25]

Our patient was having severe COVID-19 pneumonia and ARDS that required prolonged. mechanical ventilation with evidence of cytokine storm and hypercoagulability state. The course was complicated by corpus callosum hematoma, which is an uncommon site of ICH, despite the improvement in the respiratory manifestation the clinical picture became more complicated by developing pulmonary embolism.

In our patient, the endothelial dysfunction secondary to the intense inflammatory condition could explain the cerebral vessel damage causing ICH rather than a manifestation of bleeding tendency, as the hypercoagulability state resulted in PE. COVID-19 could be the umbrella under which all the events can be explained.

Reporting such a case might help to increase the understanding of the neurological complications associated with COVID-19 and will increase the awareness of possible challenges emerging while treating COVID-19 patients.

\section{Conclusion}

Corpus callosum is a rare site for intracranial bleeding complicating the course of sever COVID-19 pneumonia.

\section{References}

1. Zhou F, Yu T, Du R, Fan G, Liu Y, Liu Z, et al. Clinical course and risk factors for mortality of adult inpatients with COVID-19 in Wuhan, China: a retrospective cohort study.

The Lancet. 2020 Mar;395(10229):1054-62.

2. Ellul MA, Benjamin L, Singh B, Lant S, Michael BD, Easton A, et al. Neurological associations of COVID-19. The Lancet Neurology. 2020 Sep;19(9):767-83.

3. Wu F, Zhao S, Yu B, Chen Y, Wang W, Song Z, et al. A new coronavirus associated with human respiratory disease in China. Nature. 2020 Mar;579(7798):265-9.

4. Bao Y, Lin SY, Cheng ZH, Xia J, Sun YP, Zhao Q, et al. Clinical Features of COVID-19 in a Young Man with Massive Cerebral Hemorrhage-Case Report. SN Compr Clin Med. 
2020 Jun;2(6):703-9.

5. Baig AM, Khaleeq A, Ali U, Syeda H. Evidence of the COVID-19 Virus Targeting the CNS: Tissue Distribution, Host-Virus Interaction, and Proposed Neurotropic

Mechanisms. ACS Chem Neurosci. 2020 Apr 1;11(7):995-8

6. Wu Y, Xu X, Chen Z, Duan J, Hashimoto K, Yang L, et al. Nervous system involvement after infection with COVID-19 and other coronaviruses. Brain, Behavior, and Immunity. $2020 \mathrm{Jul} ; 87: 18-22$.

7. Li Z, Liu T, Yang N, Han D, Mi X, Li Y, et al. Neurological manifestations of patients with COVID-19: potential routes of SARS-CoV-2 neuroinvasion from the periphery to the brain. Front Med. 2020 Oct;14(5):533-41

8. Paniz-Mondolfi A, Bryce C, Grimes Z, Gordon RE, Reidy J, Lednicky J, et al. Central nervous system involvement by severe acute respiratory syndrome coronavirus-2

(SARS-CoV-2). J Med Virol. 2020 Jul;92(7):699-702.

9. Oppenheim-Eden A, Glantz L, Eidelman LA, Sprung CL. Spontaneous intracerebral hemorrhage in critically ill patients: incidence over six years and associated factors.

Intensive Care Medicine. 1999 Jan 26;25(1):63-7.

10. Carroll E, Lewis A. Catastrophic Intracranial Hemorrhage in Two Critically Ill Patients with COVID-19 [published online ahead of print, 2020 May 26]. Neurocrit Care . 2020;1-5.

11. Mao L, Jin H, Wang M, Hu Y, Chen S, He Q, et al. Neurologic Manifestations of Hospitalized Patients With Coronavirus Disease 2019 in Wuhan, China. JAMA Neurol. 2020 Jun 1;77(6):683.

12. Reddy ST, Garg T, Shah C, Nascimento FA, Imran R, Kan P, et al. Cerebrovascular Disease in Patients with COVID-19: A Review of the Literature and Case Series. Case Rep Neurol. 2020 Jun 11;12(2):199-209.

13. Sharifi-Razavi A, Karimi N, Rouhani N. COVID-19 and intracerebral haemorrhage: causative or coincidental?. New Microbes and New Infections. 2020 May;35:100669.

14. Radmanesh A, Derman A, Lui YW, Raz E, Loh JP, Hagiwara M, et al. COVID-19associated Diffuse Leukoencephalopathy and Microhemorrhages. Radiology. 2020 Oct;297(1):E223-E227.

15. Al-olama M, Rashid A, Garozzo D. COVID-19-associated meningoencephalitis complicated with intracranial hemorrhage: a case report. Acta Neurochir. 2020

Jul;162(7):1495-9

16. Wee NK, Fan EB, Lee KCH, Chia YW, Lim TCC. CT Fluid-Blood Levels in COVID-19 Intracranial Hemorrhage. AJNR Am J Neuroradiol. 2020 Sep;41(9):E76-E77.

17. Benger M, Williams O, Siddiqui J, Sztriha L. Intracerebral haemorrhage and COVID-19: 
Clinical characteristics from a case series. Brain, Behavior, and Immunity. 2020

Aug;88:940-4

18. Poillon G, Obadia M, Perrin M, Savatovsky J, Lecler A. Cerebral venous thrombosis associated with COVID-19 infection: Causality or coincidence? [published online ahead of print, 2020 May 11]. J Neuroradiol . 2020;S0150-9861(20)30167-X.

19. Coolen T, Lolli V, Sadeghi N, Rovai A, Trotta N, Taccone FS, et al. Early postmortem brain MRI findings in COVID-19 non-survivors. Neurology. 2020 Oct 6;95(14):e2016e2027.

20. Ghani MU, Kumar M, Ghani U, Sonia F, Abbas SA. Intracranial hemorrhage complicating anticoagulant prophylactic therapy in three hospitalized COVID-19 patients. J Neurovirol. 2020 Aug;26(4):602-4.

21. Solomon IH, Normandin E, Bhattacharyya S, Mukerji SS, Keller K, Ali AS, et al. Neuropathological Features of Covid-19. N Engl J Med. 2020 Sep 3;383(10):989-92.

22. Harisha PN, Reddy VU, Agrawal A, Kodali G. Massive spontaneous corpus callosal hemorrhage with intraventricular extension. roneuro [Internet]. 2014Jun.15 [cited 2020Oct.27];21(2):202-5.

23. Aji, Y.K., Apriawan, T., Bajamal, A.H. 2017. Conservative treatment of corpus callosum hemorrhage in severe traumatic brain injury: a case report. Bali Medical Journal 3(3): S97-S99

24. Connors JM, Levy JH. COVID-19 and its implications for thrombosis and anticoagulation. Blood. 2020 Jun 4;135(23):2033-40.

25. Klok F, Kruip M, van der Meer N, Arbous M, Gommers D, Kant K, et al. Incidence of thrombotic complications in critically ill ICU patients with COVID-19. Thrombosis

Research. 2020 Jul;191:145-7.

\section{Hosted file}

image1.emf available at https://authorea.com/users/357133/articles/532466-corpus-callosumhematoma-as-a-rare-complication-of-covid-19

Figure 1: MRI Brain Axial T2W image of the brain (A) shows well-defined hyperintense intraparenchymal hematoma in the splenium of the corpus callosum with mild perilesional vasogenic oedema (White arrow). Pre and post contrast axial $\mathrm{T} 1 \mathrm{~W}$ images of the brain $(\mathrm{B} \& \mathrm{C})$ demonstrate hyperintense hematoma in the splenium of the corpus callosum with no post contrast enhancement. Axial Susceptibility Weighted Image (SWI) of the brain (D) reveals blooming in the splenial hematoma as well as another haemorrhagic lesion in the genu of the corpus callosum (black arrow), there are also multiple microhaemorrhages in the cortical subcortical regions of bilateral cerebral hemispheres. Axial Diffusion-weighted image and corresponding ADC map of the brain $(\mathrm{E} \& \mathrm{~F})$ show diffusion restriction in the subacute splenial hematoma.

\section{Hosted file}


image2.emf available at https://authorea.com/users/357133/articles/532466-corpus-callosumhematoma-as-a-rare-complication-of-covid-19

Figure 2: Coronal maximum intensity projection (MIP) image of pulmonary angiogram shows embolus (white arrow) in the anterior branch of the left main pulmonary artery.

\section{Hosted file}

image3.emf available at https://authorea.com/users/357133/articles/532466-corpus-callosumhematoma-as-a-rare-complication-of-covid-19

Figure 3: A follow-up axial CT head image shows resolution of the hematoma with residual hypodensity in the splenium.

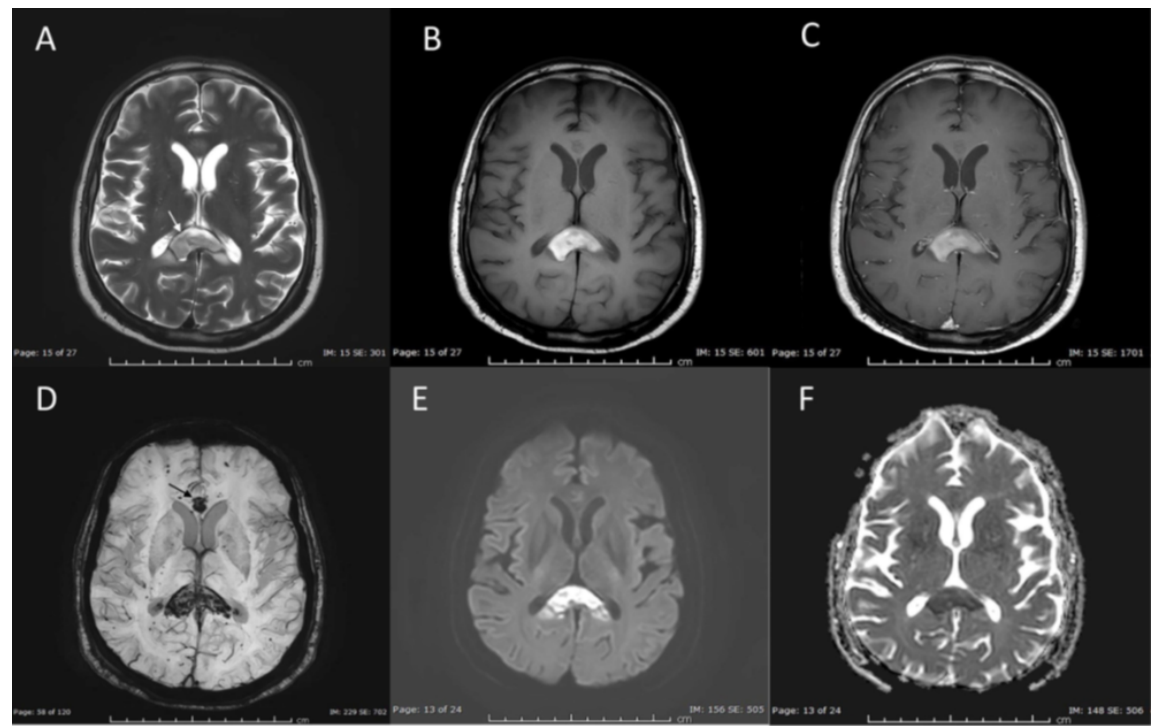




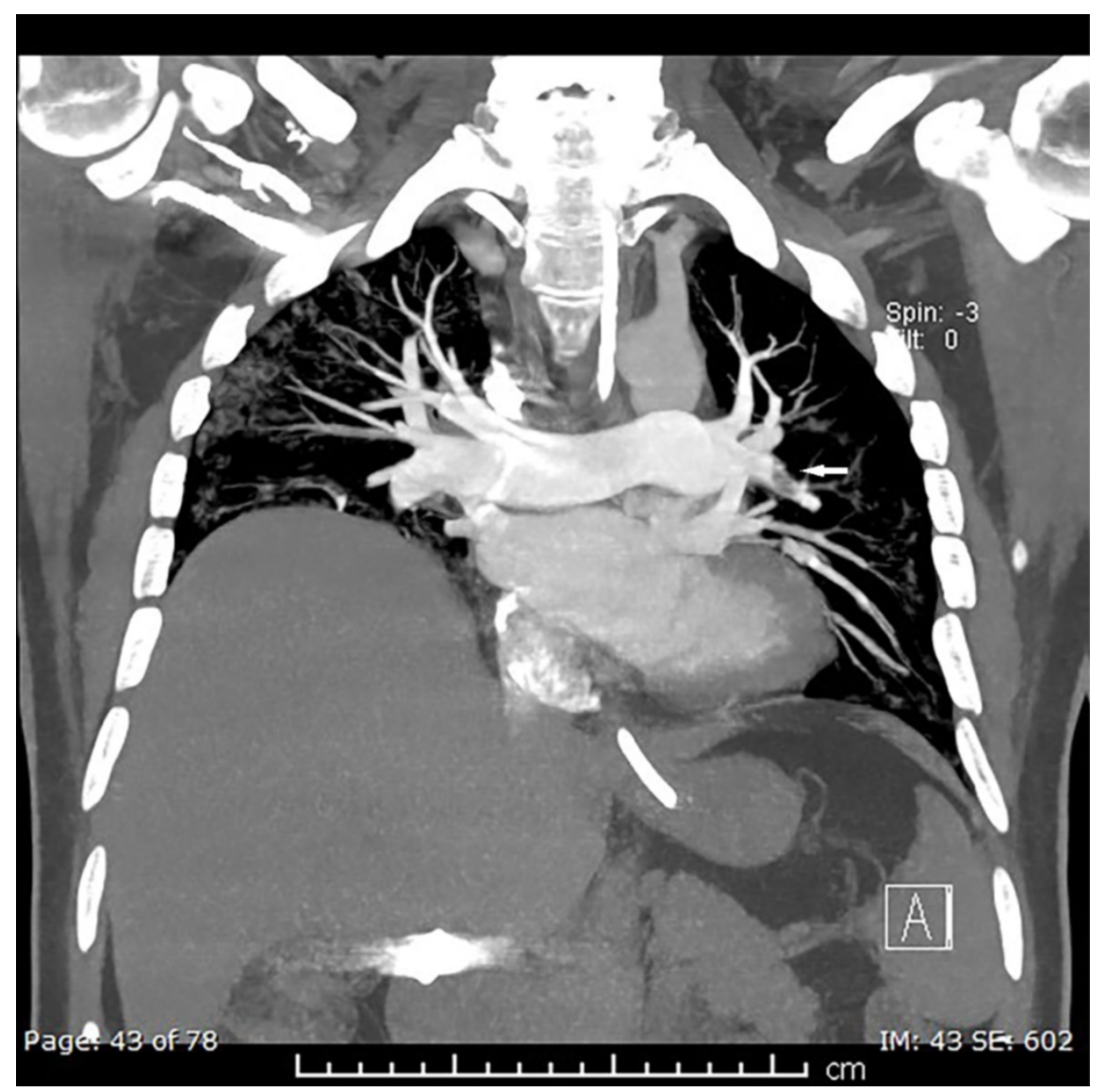




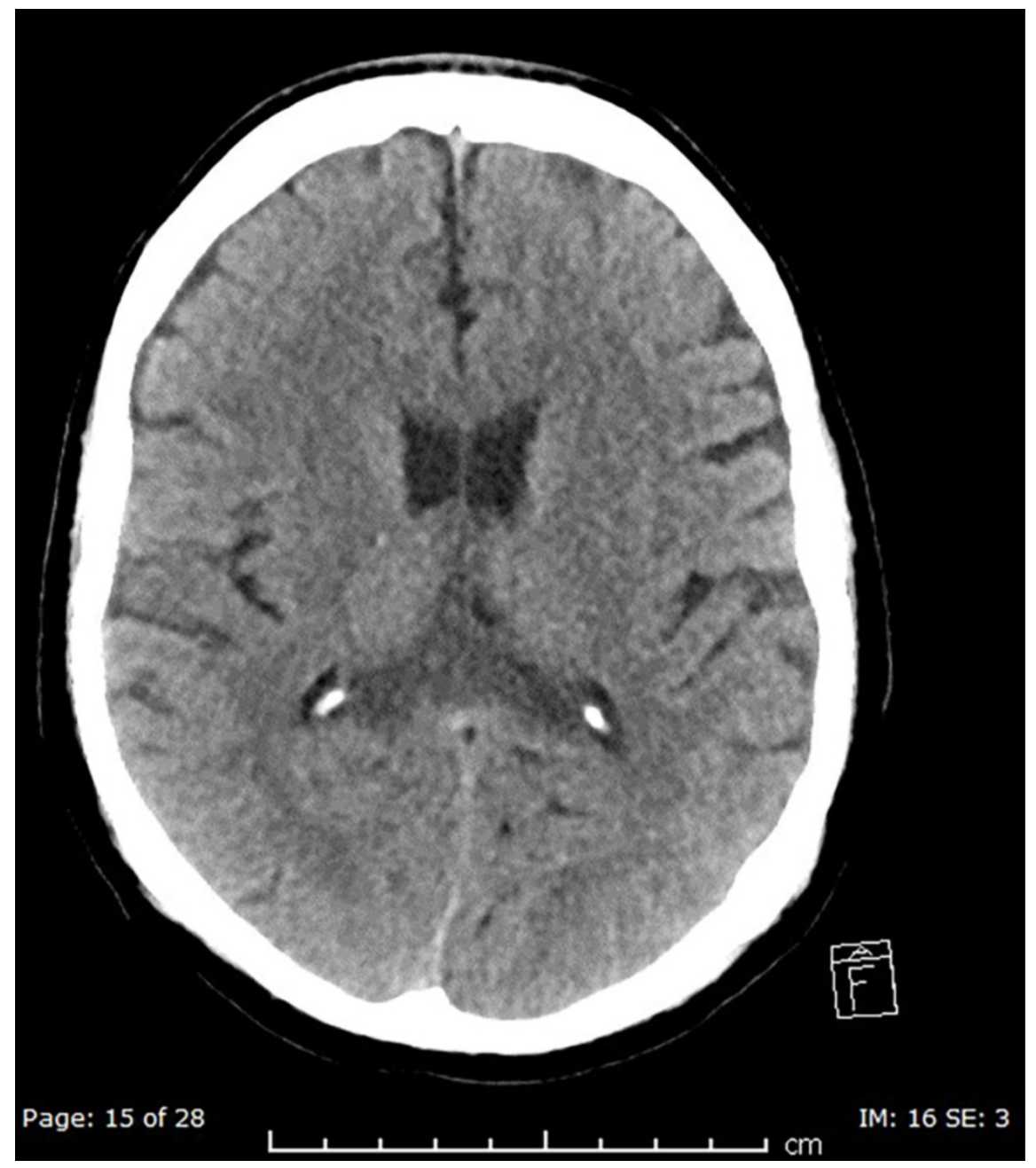

